\title{
Debate Paper \\ Privacy, data protection and freedom of information
}

\section{Will more information availability result in more satisfied customers?}

What are the relative places of legislation and self-regulation?
We decided it might be interesting to stimulate a debate on these issues. So we circulated a short series of conundrums (reproduced here as an Introduction) to three distinguished contributors, and invited them to comment freely. We then let each contributor see the other contributions, with the opportunity to respond further ...

\section{Introduction}

Consumers value something they call privacy (which is short of a precise definition). They also dislike being constantly exposed to advertising of kinds irrelevant to them (either overall, or in relation to their current needs/desires); 'junk mail' is the most frequent object of this dislike, but every advertising medium suffers to some extent. From this arises a conundrum: the greater the amount of data available to advertisers (in any medium) about individuals, the greater the reduction in their feelings of privacy, but the greater the chance of receiving relevant, and avoiding irrelevant, advertising. (That at least is the industry's official line: do we believe it? And if not, why not?) Advertisers do bring some of their troubles on themselves, but they are also exposed to the double whammy of being attacked for irrelevant untargeted advertising today, and sinister intrusions on privacy tomorrow. How do we solve this conundrum? In real terms? In PR terms? Both?

There is another polarity: the whole concept of privacy (however defined) runs counter to the idea of freedom of information - the bedrock of Karl Popper's 'open society', and enshrined in statute in the USA (although the UK, which has a much longer and more illiberal tradition of secrecy, is a great deal more ambivalent, while in much of Europe the idea is pretty well unknown). Is the invocation of freedom of information, in relation not to government decisions but to personal data for commercial use, a descent from the sublime to the ridiculous? Or is it a significant fact that in pre-1990 Moscow (no open society that) there was no public telephone directory? Is it merely paranoid to see current moves in the UK to restrict commercial usage of the electoral register as a small step, intentional or not, towards totalitarianism?

In continental Europe, with its dirigiste tradition, the answer has been that the state determines the 'rules of engagement' between advertiser and consumer: the Data Protection Directive is a prime example. The UK has followed a very similar path, but also has a powerful tradition of self-regulation, which in European countries is sometimes portrayed as a mere fig-leaf for skulduggery (and instances of that are not lacking - the venerable Law Society among others). In the USA there are no statutory rules; there are codes of practice within industry, but it is open to question how effective they are - or can be. Self-regulation lacks 


\section{Does the Internet challenge the accepted ideas of privacy and freedom of information?}

\section{How customer- centric can commerce be?}

\footnotetext{
Marty Abrams

is responsible for assuring that Experian's US business units are managing information in accordance with the company's information values. Marty is also active with government and industry groups in the discussion on balanced information management guidelines. Marty has been actively involved in business privacy issues for over 11 years.
}

the ultimate means of enforcement; legislation lacks flexibility and the ability to reflect changing circumstance.

Viewed from a static position in 1999, the European approach can seem powerful. While the US literature continues to deal in a very legalistic way with attempts to define privacy as a basic human right, and to talk about consumers contracting with advertisers to supply personal data in return for some consideration, Europe has almost bypassed the whole 'privacy' debate (and taken a lot of emotion out of the issue) by concentrating instead on a series of purely pragmatic rules about 'data protection'. However, no position is static for long. The Data Protection Directive was 20 years in the making; by the time it became effective across the EU the Internet was in full flow. So now we have advertisers in a medium which is, both intrinsically and by design, anarchic and universal, being theoretically subject to a set of statutory regulations which are geographically defined and written without reference to, or thought of, this medium. We do not have another 20 years to solve this problem; does it mean that statutory instruments are inapplicable? If so, what alternatives are there? To what extent can self-regulation be made to work, where and how?

Is it possible that this whole debate is becoming out of date? As business increasingly turns to intensive cultivation of existing customers (where these concerns are much less) rather than acquiring new customers, is it possible that these issues have lost a little/a lot of their urgency and importance? Should control of mode, channel and content of communication be in the hands of the customer (which is perhaps the tendency with the Internet) in return for data provision? How would (could) this work across all media?

\section{Response by Marty Abrams, vice president, information policy and privacy, Experian, USA}

\section{Information policy in a services-centred economy: A matter of balance}

Economic historians may look back 50 years from now and find Jacques Nasser's statement, 'I want to know the coffee pot you use and the watch you wear to produce the right car for you', as marking the global transition from a manufacturing-centred to a service-based economy. But Nasser is not an economist or marketing professor, he is the CEO of Ford. And the pronouncement was not made in a learned journal, but rather the Wall Street Journal. With that statement, Nasser demonstrated that information technology had taken traditional sectors from improving old processes to revolutionising the way managers think about their markets. In an Internet-connected world consumers call the shots, and in manufacturing companies understanding individual demand drives production. A service-based economy is not just about fast food and financial services. A service-based economy is one where all sectors take on the demand-driven aspects of individually centred services; one driven by information about the consumer. 


\section{Information technology first used to make the manufacturing process better}

\section{The application of consumer information takes us to the next level}

\section{The trust deficit}

\author{
The key difference \\ between European \\ and American \\ privacy regimes
}

In the 1980s, the auto industry used information technology to speed up the design of cars, and to schedule just-in-time manufacturing and quality control processes that allowed the industry to cut production costs and better respond to marketplace feedback about the products they supplied. These procedures brought huge financial rewards, but were just the logical conclusion of a process that began with Henry Ford when he used the assembly line to produce cars that were affordable for the working man. Manufacturing expertise led the market, with scientific-based demand modelling playing second fiddle.

Manufacturing came first, while market response came second.

As we move into the new century, manufacturing expertise is a given. If you do not make a great product that you deliver at a great price, you do not have the ticket to play. What will differentiate the winners and the losers is their ability to reap the efficiencies that come from understanding the nature of individual demands. Not only will the market make goods better, cheaper and quicker, but it will produce the right goods based on an information-driven understanding of individual consumer demand applied at an aggregate level. We will anticipate where the consumer will be, rather than responding to where he has been.

We return to Nasser's comment. He wants to know the coffee pot you use and the watch you wear so that he can produce only the vehicles you will want to buy. By doing so, he removes the inventory shocks that come from producing Edsels when the market wants SUVs. The service economy is not about fast food and financial services; the service economy is about moving from a supply-driven to a demand-driven economy fuelled by personally identifiable information. The amount and quality of that information will ultimately determine the quality of the demand predictions we make. Tools are important, but the data are critical to success. Think of it in these terms. An expensive radio will make a weak radio signal sound a little better, but not as good as a strong radio signal being received by an inexpensive radio. The quality of the signal is more important then the price of the radio. Data modelling tools are the radio, while personally identifiable data are the signal. The future quality of statistical tools is a given. The future availability of data will determine the quality of results.

At the same time, the massive application of personally identifiable information in the US economy, without appropriate use protocols that people understand, has led to a trust deficit that endangers those same flows of data. In simple terms, flexible information use is a prerequisite for creating a service-based economy, while data-subject trust is necessary for maintaining such an economy. This is a dilemma that neither Europe nor the USA has dealt with well.

No one ever bought a coffee pot or watch to prevent inventory shocks in consumer durable markets. The fact that personally identifiable information aggregated together and mined with statistical tools creates significant economic benefits is a by-product that we either choose to harness or not. Data protection as established by European law requires the parties that collect and use data to anticipate future use, and reference that use in the notices they give to data subjects. 
Data protection is based on a very simple concept: one collects information for a particular set of purposes, and one can only use the information for those or a related purpose. Any other use requires the consumer's specific consent. For Ford to use watch and coffee pot information in Europe the company needs to ensure that data were collected with notices flexible enough to accommodate that use. The European system is based on human rights conventions, and creates a higher order of legally respected privacy.

The US system is more flexible. One can use information that is collected in a lawful fashion for any purpose that:

— is not precluded by sector-specific law;

- is not harmful to the consumer;

- does not go beyond society's sense of what is appropriate.

This allows a marketer to test the predictive value of data long after they have been collected, without concern for the notice used when the data were collected.

The US system of information balance has accommodated a long economic expansion with minimal inflation. The creation of knowledge-based employment has reduced unemployment to levels not seen since the early 1960 s. 'Putting Services to Work', a paper published by the Commission of the European Union, blames economic stagnation on no growth in the services sector and no growth in knowledge-based employment. While there are many explanations for such slow growth, the fact that Jacques Nasser can more easily use information in the USA about the watch you wear and the coffee pot you use is a contributing factor. Data protection, by pre-empting secondary use of information, acts as an ongoing and slow deflator of economic growth.

As Figure 1 shows, the balance beam rests on a fulcrum. All the

Privacy/trust balance beam instruments of trust are on the left. They include:

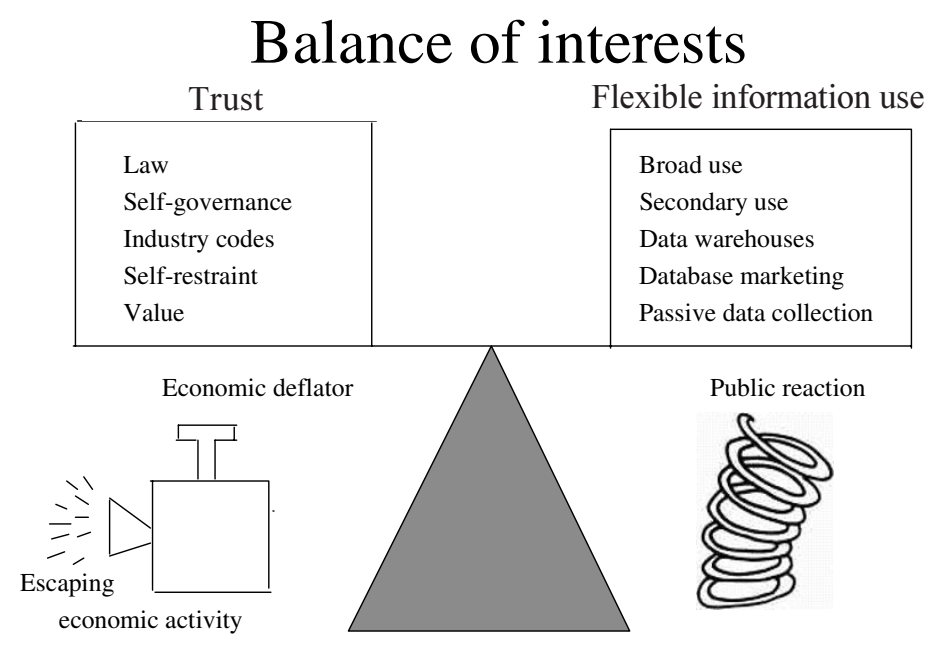

Figure 1: The privacy/trust balance beam 
- legal rights;

- industry standards with accountability;

— industry codes (voluntary, with accountability);

- corporate self-restraint;

- the value consumers receive from the use of the information.

The weight on the right includes all the processes related to using information to understand demand and apply this to the marketplace. Those processes include:

— broad market-driven uses of information;

- data warehousing;

- data mining;

- database marketing;

- file enhancement;

- customer relationship management.

As the balance beam shows, the system works best when it is balanced. When one goes too far in the use of information, and goes beyond the consumer or society's expectations:

— one tips the balance;

- the spring coils;

- the beam bounces back by putting greater weight in law, industry code, or corporate restraint;

- the economic deflator gets hit;

— economic activity - at the corporate or societal level, or both gets reduced.

On the other hand, if one puts too much weight on trust in a fashion that restrains the demand-driven service economy:

- the beam sags;

- the deflator is pushed, reducing economic activity;

— it does not self-correct.

\section{So where does that leave us?}

We have a global policy mess. The marketplace is becoming global in nature, with policy processes that could not be more different. Data protection and its severe restraints on secondary use of information have slightly depressed the economic deflator in Europe. The explosion in information and communications technology in the USA has created multiple incidents of organisations going beyond the consumer's expectation. Furthermore, technology has made it possible for smaller, privately owned organisations with limited concerns about reputational risk to exploit information. Those trends have brought out the schizophrenia of the American consumer: they want greater controls, but not at the expense of the value that comes from flexible information use. They will applaud the application of Nasser's information tools, while being critical of the data flows that make those tools possible. 
Towards a solution

\section{Afterword}

While direct marketing has grown in Europe, it has not grown at the rates seen in North America. But the growth rates in North America are not sustainable without greater public trust. Neither side of the ocean has the policy infrastructure in place for the new millennium. The solution lies in a new information policy paradigm that is neither North American nor European, but rather based on a commonality of global consumer interests.

One begins with the givens:

- the best balancers of the beam are data uses and processes that create real value for both consumer and marketers while preserving a private space for data subjects;

- value comes from flexible use that is within the bounds of consumer acceptance;

- acceptance comes from consumer knowledge and understanding of appropriate choices, and the ability to exercise those choices easily;

- marketer accountability for responsible use of information is a must.

I would suggest that, based on the parameters listed above, a grouping of thought leaders could develop that new information policy paradigm in a manner that would preserve both public trust and the processes suggested by Nasser's quote. I believe the thought leaders should drive towards high-level principles that require:

- organisations to describe the value the consumer gets from the data flows;

- organisations that collect information to be open and honest about the data they collect and their use, and organisations that aggregate information must be accessible as well;

- consumers to have information about their choices and how they can easily exercise those choices, and to be assured the choices will be honoured;

- organisations to explain how they will be accountable for the information they use;

- consumers to be reminded that change is constant and will affect the use of information, and organisations to be clear about how they explain change and let consumers exercise new choices.

These basic principles could be the starting point for a new global order that assures the data flows necessary for the new economy, in which information will be the lubricant of our digital society.

I greatly enjoyed reading the contributions from Elizabeth France and Ivan Hodac, and I read their comments in light of my position that we need an approach to information practices that is global rather than American or European. Based on their comments I would suggest the following. For us to reach our potential we need to have a global approach to privacy that operates on three axes. Those three axes are data subject value, data subject protection, and competitive business processes. 
Elizabeth France

succeeded Eric Howe as the UK's Data Protection Registrar in 1994, after a distinguished civil service career in the Home Office. Her title was changed from Registrar to Commissioner in March 2000, in line with EU practice.
The genesis of data protection was growing concern in North America and Europe about surveillance in the late 1960s. The concern was government uses of information from increasingly complex databases. The issue was not balance but rather protection, and the starting point of policy was protection.

In the USA, we applied the concepts of data protection only to the government. The business focus was on the competitive process axis, unless consumer harm forced a different analysis.

In Europe many countries enacted laws applying data protection to the private sector, with the focus on the protection axis. Balance, in Europe, came from government-sponsored exceptions and interpretations that came from the data protection authorities. Balance in the USA came from checks and balances.

The growth of the Internet and Internet technologies creates different challenges for the two continents. In the best of worlds the challenges would lead to a harmonisation that would benefit the global economy.

I would suggest the USA needs norms that are responsive to surveillance, while Europe needs a process where changed use of information is less dependent on the government. One step towards moving in that direction would be the simple addition of a new information value: balance of interests. The application of that principle would move parties to start thinking on all three axes.

\section{Response by Elizabeth France, UK Data Protection Commissioner}

The French do not have a direct translation of the word 'privacy', they talk of 'la vie privée'. Perhaps that is a better starting point for discussion? It is that use of language which is picked up in my office's current mission statement: 'We shall promote respect for the private lives of individuals and in particular for the privacy of their information ...' It gives prominence to the importance of respect for private life which has resonance with Article 8 of the European Convention on Human Rights. That is important. Whether 'privacy', 'data protection', or 'fair information handling' is the term of choice, there is a danger that our background will determine the scope of what we understand. It has to be unpacked. For us the Council of Europe Convention, Treaty 108, and the OECD Principles provide the broad explanation of just what we are talking about.

The Data Protection Directive 95/46/EC provides the latest harmonised and binding framework for Europeans, explaining data protection in terms of rights to privacy and providing an enforceable code of fair information-handling practice. It makes it clear that data protection is an aspect of the fundamental right to privacy. Not an absolute right. It must be balanced. One of those balances recognises the importance for trade of information exchange. The need for balancing judgments is clear whenever we look at rights in this area.

This can present conundrums, but I do not think this would be a fair description of the situation we find in looking at direct marketing.

\section{The concept
of choice \\ The concept
of choice}

\section{Protection Directive}




\section{Freedom of information and data protection}

\section{Personal data in published lists}

One-to-one marketing and, more recently, permission marketing are embraced by the industry. Surely these approaches to marketing recognise that if I do not want Jacques Nasser to know what coffee pot I use, or what watch I wear, then that information should not be available to him. He may not then produce the car I want, but that is my business. So long as the concept of choice is inherent in these modern developments in thinking about the service economy, then they sit very well with data protection requirements. Tell the customers what you are doing. Allow them to say no. Targeted marketing with respect for the customer. Individually centred services driven by the consumer.

To give individuals that control does not run counter to arguments for open government. It may be historically true that post-war Europe has been more concerned to see personal data protected than to give emphasis to freedom of information legislation, but the importance of information in today's world will mean the principles of both will need to be acknowledged. Freedom of information is about creating open and accountable public authorities by giving citizens a right of access to the information the authorities hold. While it is the more prominent of the two rights in the USA, it is wrong to conclude that it is unknown in Europe. France, Sweden, the Netherlands, Ireland and Germany all have access legislation. They all also have well-established data protection law. Each of their data protection laws sees subject access - the right of individuals to see anything held on computer on them - as a key right. This right is to be extended to certain manual records in 2001 by virtue of the directive. Both freedom of information and data protection laws thus seek to ensure as much openness as possible. But a civilised society must also allow its citizens space. It must protect the information they have provided to the state from the eyes of others, except when there are clear grounds for overriding that right to privacy. So an area of tension between the two may arise where one individual seeks personal information about another. Where the line is drawn will vary, but UK law is likely to make clear that where personal data are sought they can only be provided to third parties if the requirements of the Data Protection Act can be met.

The control of personal data in what have been traditionally published lists is a different issue. Some lists are published under statute for a particular reason: lists of doctors so we can check credentials; lists of shareholders for publicly quoted companies; lists of electors to ensure fair elections. However, modern technology's ability to mix, match and merge these lists has led to large numbers of people finding themselves, often unknowingly, supporting one of the basic principles of data protection, the principle of 'finalite'. They do not like the fact that information obtained for one purpose is being used for another. Over 50 per cent of subscribers have chosen to take their names and numbers out of the phone book. There is no such choice in the case of the electoral register without risk of criminal penalty, but there are significant numbers who are 'disappearing' because they do not like the consequences of secondary use. The government's current proposals on control of the electoral register are made in the light of the Human 


\section{Self-regulation}

\section{A combination of approaches}

\author{
Privacy and \\ democracy in \\ cyberspace
}

Rights Act and the Data Protection Act. They are designed to ensure that, having obtained information using the coercive powers of the state, they allow choice over further uses which go beyond the state's legitimate need for the information. Empowering the citizen in this way is the antithesis of totalitarianism.

If we are to make progress we must move away from stereotypes. While it is true that the USA has no federal-level law relating to data protection in the way that Europe does, last year more than 7,000 pieces of privacy law were making their way through US state legislatures. It is equally true that not all aspects of the law in Europe are enforced with equal vigour in each of the member states. Meanwhile, in Europe the self-regulatory approach, through, for example, the encouragement of standards, is widespread. It is in this area that codes of practice such as that proposed by FEDMA are not only welcomed but encouraged by the directive itself. The emphasis is different. The starting points are different. However, with some willingness to look broadly we should be able to find the commonality necessary for consumers to be confident when trading in different jurisdictions. It is interesting to look beyond Europe and the USA to the picture which is emerging in the AsiaPacific region. Nigel Waters, privacy consultant, in his paper to the International Conference on Data Protection and Privacy Commissioners in Hong Kong last September, set out a third way and challenged the polarisation which has tended to characterise debate. ${ }^{1}$

We must recognise the need for a combination of approaches: law; codes of practice; uses of privacy-enhancing technology; and consumer education if commerce is to be able to retain consumer confidence in the information age.

The need for a combination of approaches becomes even more apparent when we look at the challenges presented by the Internet. Data protection law is not technology specific and the principles it lays down are as relevant for cyberspace as they are to other processing of personal data. There is no virtue in seeking technology-specific legislation since the gestation period of legislation will never match that of emerging technology. It is therefore preferable for the law to provide a high-level framework - to provide the penalties where abuse occurs. The challenge of cyberspace relates to the need to identify a jurisdiction, but reliable players can assist by making clear under what laws they operate. Here the privacy statement has a major role to play. The OECD pilot on privacy statements is proving valuable; excellent statements are appearing on sites with worldwide origins. Audit bodies are becoming established to check that sites are living up to the claims they make. We and others in similar roles are encouraging individuals to check for such statements and look to see if they are happy with what is offered before doing business. The combination of law, self-regulation, technology and education really comes into its own in this environment.

There is a long way to go. The American academic Paul Schwartz, in a recent paper; concludes that we need 'multidimensional rules that set out fair information practices for personal data in cyberspace'. He suggests four requirements: 
'(i) defined obligations that limit the use of personal data;

(ii) transparent processing systems;

(iii) limited procedural and substantive rights; and

(iv) external oversight. ${ }^{2}$

He argues that these require enactment of federal law in the USA. Whether or not that view is accepted, we all need to play our part in ensuring that there are accepted standards for the processing of personal data in cyberspace.

If business fails to recognise the importance of these issues, customer confidence, already fragile, will collapse and the full potential to exploit cyberspace in the consumer marketplace will be unrealised. My concern is with individuals, whether customers, employees or citizens. Respecting their information will accord to them the respect which will give them the confidence to trade. The more control they can have over the use of their data the better. Data are not, however, a commodity in themselves, to trade as other goods are traded. In the virtual world information about us provides our image, our profile, more clearly than in the past. It is an interesting idea that the individual is, in cyberspace, moving out of urban anonymity and into something closer, at least in terms of what those trading with us know about us, to the traditional village. Village confidences were, however, shared on a solid foundation of mutual trust. The new foundation is not yet deep enough or strong enough. Far from hindering its establishment, data protection principles form an essential part of the structure. Our rights in relation to our own information are increasingly important. Anyone who uses it must recognise the obligations that use places upon them.

\section{References}

1. Waters, N. (1999) 'Re-Thinking Information Privacy - A Third Way in Data Protection?', paper presented at the 21 st International Conference on Privacy and Personal Data Protection, Hong Kong, 13-15 September 1999, Conference Proceedings, Office of the Privacy Commissioner for Personal Data, Hong Kong, pp. 49-59.

2. Schwartz, P. M. (1999) 'Privacy and Democracy in Cyberspace', Vanderbilt Law Review, Vol. 52, No. 6, November.

Ivan Hodac is FEDMA chairman, and senior vicepresident of Time Warner Europe, Belgium. His responsibilities include coordinating the public affairs of all Time Warner operating divisions and representing Time Warner interests vis-à-vis international organisations and institutions, in addition to EU governments.

\section{Response by Ivan Hodac, Senior vice president, Time Warner Europe}

The debate on privacy and protection for individuals' data has been rumbling around on both sides of the Atlantic for over a generation now. Recently, due to the new e-commerce developments, the implementation of the EU's 1995 Data Protection Directive, and the EU/US negotiations on a 'safe harbour' agreement, data protection has again hit the headlines.

I do not feel that these 'new' developments have brought any very revolutionary ideas to the debate, but they have certainly concentrated minds again on one of the most controversial and vital aspects of data privacy - the 'balance' of interests between the individual citizen on 
US laws more restrictive than European legislation the one hand, and state and commerce on the other hand, in their desire to hold, process and use personal data.

This balancing act is based on completely different criteria for the state, which claims the need to hold data for a vast array of different reasons (tax, health, voting, licensing cars, TVs, hunting/fishing, etc, property, and so on), and commerce, which needs information on both existing customers, in order to serve them better, and potential customers, in order to market to them.

One of the underlying problems, in my view, is that both state and commercial use of data were covered from the very beginning of the debate by the same regulations. This muddled the purpose of the laws, and allowed the regulators in Europe to squeeze themselves off the public data debate hook by concentrating on the need to control commerce's use of personal data. In the USA, on the other hand, there are a number of federal laws on data privacy, almost all of which deal specifically with the use of personal data by the state. The US administration has been quick to point out a number of times during the recent discussions on the EU Data Protection Directive that, when it comes to the citizen's protection against the collection, processing and use of data by the state, the US laws are more complete and more restrictive than the European regulations.

There is also a secondary reason why publicly acquired and used date should be, in my view, separated from commercial use. The use of public data by commerce is being increasingly restricted (for example, the current practice in the UK of using the electoral roll to verify the most up-to-date basic information on citizens, such as addresses, change of name, etc, is now likely to be stopped). At the same time, there have been some unfortunate experiences involving the misuse of public data. One example that springs to mind is the use of that very same electoral roll in Scotland by local authorities to check those eligible for local taxes in the 1980s.

The need to balance interests has also certainly been thrown into focus by the developments in e-commerce. All research surveys have found that the majority of consumers, on both sides of the Atlantic, would favour greater protection from the indiscriminate use of personal data, and, in particular, from the covert collection of data on the Internet. Data privacy and security for financial transactions are the two issues most referred to by respondents to e-commerce surveys.

Industry has responded by introducing ways to protect users through privacy policy programmes and e-mail preference ('do not contact') systems, particularly in the USA. The US Direct Marketing Association's 'Privacy Now' programme, for example, requires all the US DMA's members to have an active privacy policy, and it has pledged to disbar members that do not comply. In November it threw out two members who had refused to introduce effective systems on their websites, and it is presently reviewing other cases. The Federal Trade Commission has supported the US DMA's programme, and can impose injunctions against companies which ignore the self-regulation codes. 


\section{FEDMA is launching Europe-wide programme to provide trust and confidence in online marketing}

In Europe, the EU's Data Protection Directive requires that citizens have the right to opt out of marketing lists, and to be clearly informed if data are collected from them and for what purpose(s) these data will be used. Thus, in theory, the citizen should be adequately protected and able to reject the use of his/her data for direct marketing purposes on either side of the Atlantic. However, clearly this message has not adequately reached the citizen or business in Europe. In a study by the Spanish E-Commerce and Direct Marketing Association (FECEMD), a number of sites directed at Spanish users did not carry privacy statements, whereas the same sites directed at US users carried privacy policy statements.

Partly this is explained by the different approaches of self-regulation, which must be transparent in order to work, and the law, which is often naively considered to be obvious and therefore not to need explanation. There is probably no doubt that these Spanish sites applied the (rather restrictive) Spanish data protection law. However, as the FECEMD pointed out, this is not going to assure the citizen/user who reads articles in the press about the dangers of data abuse on the Internet. The FECEMD has therefore introduced a trustmark supported by a privacy policy statement to help to reassure users.

FEDMA is also launching a Europe-wide programme designed to provide trust and confidence for the use of online marketing, the 'Ring of Confidence', which will include a privacy policy, a code of good business practices, consumer complaint mechanisms, links to both verification and authenticity programmes, an e-mail preference service and out-of-court arbitration systems. FEDMA is currently looking for industry funding for this programme.

The FEDMA code will be divided into two parts: a series of rules on good business ethics, based on the EU Distance Selling Directive; and a data privacy policy statement based on a code of practice presently being negotiated between the national data commissioners (with European Commission support) and FEDMA.

This exercise is very interesting for two reasons: first, because it will be the first sectoral code agreed under the Directive, which specifically invites sectors to negotiate codes with the collective body of national data commissioners (the so-called Article 29 Group); and second, as a result, FEDMA's privacy policy will therefore become the officially recognised European system.

There is a very good set of precedents for codes of conduct and data protection in Europe. The present Dutch law, which is currently under review, specifically leaves detailed rules for sectors to negotiate codes of practice. Direct marketing and data protection codes also exist in many other countries - France, Portugal, Spain, the UK and Ireland, for example, all have well-developed codes which are recognised by the national authorities and applied energetically. In a recent speech to a FEDMA seminar on data protection, the European Commission recognised that there are already considerable problems in the national application of the Data Protection Directive (only eight countries have so far fully implemented the Directive, although all the 15 member states were supposed to have done so by October 1998). The 


\section{Self-regulation is the solution}

Commission suggested that codes might be one of the most effective ways to prevent different interpretations of the Directive, which is certainly a novel approach for legislators.

Data protection and the rights to privacy of the individual citizens are in the interests of both consumers and industry. This is clearly a common interest, although many commentators may not recognise (or wish to recognise) the fact.

Furthermore, the whole debate on privacy must be considered in new terms: we have lived through a period of mass production and mass anonymity with super - and mega - markets catering to the masses, and mass media advertising to the masses. This is changing: the Internet and database marketing allow us to identify customers and tailor our offers to their needs and aspirations. The question is, can the individual citizen make the leap from being treated by business as one of a mass to being treated as an individual (albeit not always as yet with great subtlety or finesse)? In the past this individual approach was the way of the village, where the postman, butcher, baker and other tradesmen knew their customers' interests, preferences and buying habits.

Can we, urban animals that we have now become, accept a more individualistic service from our suppliers? I believe we can - and will - if the conditions are right and there are suppliers/marketers who promote trustworthy e-commerce. We must be particularly sensitive, awake to the potential concerns of our customers and willing to remedy any problems rapidly in this new marketing environment. In other words, if we expect customers to interact with us, then we must interact with them!

Everyone agrees that we cannot expect a global law that would regulate the use of personal data on the Internet. Self-regulation is therefore the solution. It will need other applications to gain the e-commerce users' trust and confidence. The use of trustmarks and software solutions ('privacy enhancing technologies' or PETs) will separate the good from the bad.

In my opinion, in a few years there will be more protection of data online than offline, thanks to the commonly recognised need to gain users' confidence. I would go further, and say that if we do not achieve this goal we shall never see the full potential of e-commerce, either in Europe or globally. Certainly this is the view of the Global Business Dialogue on E-Commerce (GBDe) which I am also closely involved in, and of other business and consumer groups. Evidence of the concern and interest of everyone is not difficult to find - the Consumers International report of September, for example, or the work of the OECD, and of the consumer/industry dialogue on e-commerce. 